\title{
La práctica de karate-do y la habilidad motriz en niños con discapacidad intelectual.
}

\section{The practice of Karate-Do and motor skills in children with intellectual disabilities.}

Cuzco Yamasca María Angelica. ${ }^{1}$, Rodríguez Muñoz Solange Stephanie. ${ }^{2}$ \& Caicedo Merizalde José Guillermo ${ }^{3}$

\begin{abstract}
Introduction. Children with intellectual disabilities present problems in the development of basic motor skills, negatively influencing the inclusion processes. Considering the benefits that sports practice has for the achievement of their skills and especially KarateDo, the research is aimed at. Objective. To determine the influence of the practice of Karate-Do on the development of motor skills in children with intellectual disabilities, from the Centro de Desarrollo Integral el Niño Fundación Tierra Nueva. Methodology. The research was of a correlative, quasi-experimental type, accompanied by theoretical and empirical methods. The sample consisted of 28 children, with intellectual disabilities, to whom the Ozeretski Guillman Test was applied in two pretest and posttest moments. Determined the results of the pretest, 4 weeks of intervention were carried out by applying a Karate-Do Session Program, using the Kihon technique. Subsequently, he made a second measurement (post-test), to assess the development achieved in the following skills: Oculo-manual coordination, general dynamics, postural control and balance. Results. 1. It was demonstrated through the application of the Student's t test that when comparing the results derived from the initial and final measurements, there was a significant difference in the means of the levels achieved in the motor skills of the children. 2. It is appreciated that the applied program had very positive effects on the development of motor skills of students with intellectual disabilities, with special interest in children with Down syndrome. Conclusions. The effectiveness of the program carried out through the practice of Karate-Do based on the Kihon technique as a viable alternative

\footnotetext{
${ }^{1}$ Universidad de las Fuerzas Armadas. (ESPE), Carrera: Pedagogía de la Actividad Física y Deporte correo. macuzco2@espe.edu.ec ORCID ID: 0000-0002-0494-6464

${ }^{2}$ Universidad de las Fuerzas Armadas. (ESPE), Carrera: Pedagogía de la Actividad Física y Deporte correo ssrodriguez4@espe.edu.ec ORCID ID 0000-0002-5546-1344

${ }^{3}$ Universidad de las Fuerzas Armadas. (ESPE), Carrera: Pedagogía de la Actividad Física y

Deporte correo jgcaicedo@espe.edu.ec ORCID ID: 0000-0001-5772-3304
} 
for the integral development process of children with intellectual disabilities, with an emphasis on their motor skills.

Key Words: Intellectual disability, motor skills, Karate-Do, Kihon.

\section{Resumen}

Introducción. Los niños con discapacidad intelectual presentan problemas en el desarrollo de las habilidades motrices básicas, influyendo negativamente en los procesos de inclusión. Considerando los beneficios que tiene la práctica deportiva para el logro de sus habilidades y en especial el Karate-Do, la investigación tiene como Objetivo. Determinar la influencia de la práctica del Karate-Do en el desarrollo de las habilidades motrices en niños con discapacidad intelectual, del Centro de Desarrollo Integral el Niño Fundación Tierra Nueva. Metodología. La investigación fue de tipo Cuasi experimental de orden correlativa, acompañada de métodos teóricos y empíricos. La muestra estuvo integrada por 28 niños, con discapacidad intelectual, a los cuales se les aplicó el Test de Ozeretski Guillman en dos momentos pretest y postest. Determinados los resultados del pretest se realizaron 4 semanas de intervención mediante la aplicación de un Programa de Sesiones de Karate-Do, utilizando la técnica del Kihon. Posteriormente realizó una segunda medición (postest), para valorar el desarrollo logrado en las habilidades siguientes: Coordinación óculo -manual, dinámica general, control postural y equilibro. Resultados. 1. Quedó demostrado mediante la aplicación del T de Student, que al comparar los resultados derivados de la medición inicial y final existió una diferencia significativa en las medias de los niveles logrados en las habilidades motrices de los niños. 2. Se aprecia que el programa aplicado tuvo efectos muy positivos en el desarrollo de las habilidades motrices de los estudiantes con discapacidad intelectual, con especial interés en los niños con Síndrome de Down.Conclusiones.Se evidenció la efectividad del programa realizado mediante la práctica de Karate-Do basado en la técnica del Kihon como una alternativa viable para el proceso de desarrollo integral de los niños que presentan discapacidad intelectual, con énfasis en sus habilidades motrices.

Palabras Claves: Discapacidad intelectual, habilidades motrices, Karate-Do, Kihon,

\section{Introducción}

Durante el proceso de desarrollo evolutivo del niño este va experimentando nuevos saberes cognitivos, afectivos, del lenguaje y por supuesto motriz; esto lo logra mediante la exploración del medio que le rodea para lo cual le resulta imprescindible moverse. En este amplio y complejo proceso, las Habilidades Motrices Básicas, ocupan un lugar fundamental; precisamente por todo lo que aportan al desarrollo integral del infante.

Tomando en cuenta que debe existir una respuesta motora estas habilidades son entendidas como una capacidad natural que se dan dentro del propio desarrollo humano. Las mismas son irremplazable para la vida, implicando varios movimientos tales como: 
correr, saltar, lanzar, atrapar, reptar. Unido a esto en la propia actividad cotidiana se van perfeccionando estos movimientos permitiendo que los niños aprendan y se desarrollen, ejemplo la participación en los juegos, la realización de las tareas de la casa, subir las gradas entre otros, implican siempre el desarrollo motriz del niño.

Considerando lo explicado se reconoce su valor no solo dentro del ámbito deportivo sino para la vida en general, con especial interés en la infancia. De ahí que lograr un desarrollo óptimo de las habilidades motrices básicas es fundamental para el desarrollo general del niño, independientemente a su condición. Si desde la infancia los dotamos de todas estas herramientas contribuiremos favorablemente al logro de un aprendizaje motor más complejo.

En el caso de los menores que presentan una necesidad educativa especial, asociada a una discapacidad intelectual lo anterior adquiere mayor valía, debido a que en muchos casos puede afectarse grandemente su desarrollo, precisamente por presentar lentitud en su progreso motor. Por lo cual es necesario buscar alternativas que contribuyan al crecimiento motriz de los niños con discapacidad intelectual. Partiendo de lo planteado y teniendo en cuenta la utilidad y beneficios de la práctica deportiva para los procesos de inclusión y desarrollo de este grupo de niños, la investigación que se presenta tiene como objetivo: Determinar la influencia de la práctica del Karate-Do en el desarrollo de las Habilidades Motrices en niños con discapacidad intelectual, del CDI

\section{Discapacidad Intelectual y Habilidades Motrices Básicas. Su importancia en el desarrollo integral de los niños.}

La Organización de las Naciones Unidas ONU, (2018), revela que la discapacidad "incluyen a aquellas personas que tengan deficiencias físicas, mentales, intelectuales o sensoriales a largo plazo que, al interactuar con diversas barreras, puedan impedir su participación plena y efectiva en la sociedad, en igualdad de condiciones con las demás". Por otro lado, la UNESCO, (2004) define a la discapacidad como aquellas "limitaciones personales que implican una desventaja sustancial para funcionar en la sociedad; desventaja, que debe ser considerada a la luz del contexto ambiental, los factores personales y la necesidad de apoyos individualizados".

La Asociación Americana de Discapacidades Intelectuales y del Desarrollo (AAIDD, CREENA, 2010), manifiesta que la discapacidad intelectual, se caracteriza por limitaciones tanto en la parte intelectual, como en la conducta adaptativa. Esta discapacidad según Luckasson, et al., (2011), tiende a originarse antes de los 18 años, y puede clasificarse en leve, moderada, severa y profunda, por ende, concordamos con el autor mencionado en que en su generalidad presentan deficiencias en el desarrollo de las Habilidades Motrices. Es por ello que se requiere de atender y estimular su desarrollo desde edades tempranas.

Lucea (1999), señala que el aprendizaje de las habilidades motoras genéricas inicia en el nacimiento y continúa durante la vida del ser humano, mientras que, según Guthrie, (2015), las habilidades motrices básicas ayudan a desarrollar habilidades motrices mas 
complejas.

Lo cierto es que las habilidades motrices, son fundamentales en la vida del ser humano. En el caso de los niños que presentan discapacidad intelectual estas habilidades son afectadas considerablemente, debido a que se caracterizan por limitaciones notables tanto en el funcionamiento cognitivo, motriz, socio afectivo y del lenguaje, llegando incluso según sea su nivel de afectación al comprometimiento de su conducta adaptativa y social.

Las personas con discapacidad intelectual presentan un déficit en el desarrollo de las Habilidades Motrices en especial en la coordinación, el cual es un tema tan amplio en cuanto a su estudio por lo que varios autores la pueden definir de diferentes maneras, Rigal (2006), define a la coordinación como "una adecuada organización espaciotemporal del cuerpo en el espacio, que tiene como resultado el de alcanzar el objetivo propuesto" por otro lado los autores Schuba \& Hafelinger, (2010) manifiestan que la coordinación "comprende una acción relacionada entre el sistema nervioso central que cumple la función de órgano regulador y el de la musculatura como un órgano efector dentro de la ejecución de un gesto motor." Señalando que "La coordinación puede ser considerada como un factor primordial dentro de capacidad de rendimiento motor, puesto que su efecto es el que permite que las demás características motoras básicas de la condición física, como la fuerza, la resistencia, la velocidad y la movilidad, puedan ser utilizadas" (Schuba, 2010, pág. 7).

Por su parte la Coordinación Dinámica General comprende la capacidad que tiene el ser humano para realizar movimientos con todas las partes del cuerpo de una manera armónica y adaptada a las diferentes situaciones en las que se encuentra. Según Molina, (2011), se trata de un movimiento donde intervienen varias fracciones musculares como las extremidades superiores, inferiores o simultáneamente. Esta se basa en el movimiento con desplazamiento de cuerpo en uno o ambos sentidos, los cuales pueden ser rápidos o lentos”. Contreras, (2011), también está relacionada con el correctofuncionamiento del Sistema Nervioso Central y el musculo esquelético que se encuentra en movimiento y su mayor característica es que existe una gran participación muscular.

Para Arteaga, Ramírez \& Luna, (2020), la coordinación ojo-mano, es una habilidad cognitiva, que permite hacer actividades para las cuales usamos los ojos y las manos, en donde los ojos fijan una meta y las manos realizan las acciones para cumplir con el objetivo.

Como puede apreciarse los posicionamientos teóricos en relación con la discapacidad intelectual y las Habilidades Motrices Básicas datan desde años anteriores, en todo los aportes se puede evidenciar la importancia del desarrollo motriz para el logro del progreso integral del niño con discapacidad intelectual, sin embargo esto no siempre resulta sencillo; se requiere del desarrollo de diferentes estrategias didácticas metodológicas y en especial de las adaptaciones correspondientes.

En este orden de ideas resulta interesante valorar la práctica deportiva como vía para estimular el desarrollo integral del niño con discapacidad intelectual. Nótese que una 
alternativa viable para compensar las posibles limitaciones se encuentra en la Actividad Física Deportiva Adaptada, la cual posibilita mediante los diferentes niveles de ayudas y adaptaciones la realización de actividades favorables a incentivar el desarrollo motriz de los niños que presentan discapacidad intelectual. Diversas investigaciones han demostrado que es necesario este tipo de prácticas para mejorar su desarrollo tanto social, físico como psicológico, de ahí que sea considerada una herramienta importante dentro del proceso de intervención con los niños que presentan esta discapacidad. Ello nos hace concordar con Arana, (2017), al expresar que a mayor déficit intelectual y menor estimulación, mayores serán los trastornos motores.

\section{Un acercamiento a las características del Centro de Desarrollo Integral y a la importancia de la Investigación desde la práctica del Karate Do.}

El Centro de Desarrollo Integral El Niño, Fundación Tierra Nueva, está integrado por una diversidad de niños, todos con presencia de diferentes discapacidades. En el mismo se desarrolla un proceso de atención pedagógica basado en la integración, inclusión a la diversidad, con énfasis en la inclusión de las características y potencialidades de los niños que lo integran y de sus familias. En la investigación que se presenta nos enfocamos en el caso de los niños portadores de una discapacidad intelectual, con especial interés en los que presenta Síndrome Down, precisamente por la urgente necesidad de buscar solución a sus dificultades motrices.

La investigación busca demostrar la importancia del uso de la práctica del Karate Do, como un medio para mejorar las habilidades motrices en los niños con discapacidad intelectual, del Centro de Desarrollo Integral El Niño, Fundación Tierra Nueva. Basado en la participación directa y activa, así como en la implementando actividades lúdicas, deportivas, respaldados por una metodología de enseñanza innovadora y adaptada a las necesidades de los casos participantes, permite aportar favorablemente al desarrollo de las mismas de los niños participantes, evidenciándose los beneficios que brinda la práctica deportiva en el desarrollo optimo de los niños que presentan discapacidad intelectual.

En la actualidad la práctica de las artes marciales ha tomado gran relevancia en cuanto a la práctica a nivel mundial tanto así ha sido la aceptación de esta disciplina que se ha posesionado en el segundo deporte más practicado a nivel mundial desde que el Judo fue incluido como deporte olímpico en Tokio 1964 y el reciente ingreso del karate Do en los Juegos Olímpicos Tokio 2021.

Las artes marciales fueron clasificadas según diversos criterios de práctica y técnica, Terry, (2006) señala que " que es importante considerar las que características de todos los grupos se ven agrupados en mayor o menor proporción entre todos los estilos": los cuales se clasifican por sistemas de la siguiente manera:

- Técnica del golpe: (Wushu, Karate)

- Técnica del agarre y proyección (Judo, Aikido)

- Técnica basado en la utilización de instrumentos (Kendo, Hamkumdo) 
- Técnica basada en la conservación de la salud (Taichí, Qigong)

Las artes marciales fueron divididas en internas y externas, las internas hacen referencia al cultivo de la energía corporal y regulación de la postura la cual brinda efectos beneficiosos para la salud, mientras que las externas en el uso de la fuerza contra un objeto externo. (Soto, 2019). Manifiesta que "El Karate representa para quienes lo practican un deporte lo multidisciplinario y al mismo tiempo con grande beneficios a nivel mental y físico teniendo como objetivo que sea practicado por todos y todas sea cual sea su condición física o mental".

Por otro lado el karate es considera más que un deporte, una disciplina donde las personas que lo practican no solo descargan adrenalina sino más bien existe una fundamentación fisiológica que se encuentra basada en la enseñanza de valores como por ejemplo, el respeto mutuo, el honor, solidaridad, autocontrol, responsabilidad, puntualidad y el control de sus emociones, valores que son fundamentales en la primera infancia para con ello llegar a la adolescencia y pubertad con mayor integridad y conciencia de uno mismo. El karate en estas personas que cuentan con un grado de discapacidad, como la discapacidad intelectual, permitirá una adaptación al entorno disciplinario y esto dará un mayor desarrollo de las habilidades motrices con mayor facilidad y esto llevará a un proceso de aprendizaje consciente en los diferentes escenarios de competencia como de entrenamiento acrecentando sus experiencias motrices.

Existen elementos que se favorecen a través de la practica del arte marcial, como también componentes coordinativo psicomotriz (Kase, 2014)

- Ayuda en el desarrollo óptimo de la coordinación motriz.

- Favorece aun buen desempeño en las destrezas reflejo y reacciones en las diferentes etapas.

- Permite liberar energía en los niños incrementado la concentración.

- Fomenta valores y principios en los infantes.

- Incrementa el autocontrol, disciplina y respecto en su entorno y con los suyos.

- Desarrolla capacidades físicas y sus diferentes habilidades motrices.

En varios estudios revisados por la académica The Sport Journal norteamericana, menciona algunos elementos que favorecen con la practica del Karate Do al desarrollo de la habilidad motriz (Fuentes, 2016).

- Cuenta con grandes beneficios sobre todo en los niños, en sus capacidades físicas tanto coordinativas como condicionales y flexibilidad.

- Con los sinnúmeros de ejercicios que se practican en este deporte se ha observado que la postura y los movimientos corporales también son estimulados.

- Tiene gran impacto en la estimulación sensorial de mayor velocidad y reacción como también incidencia en el campo intelectual. 
Como arte marcial mediante el cual se puede adquirir muchas habilidades como lo menciona (Soto, 2018), al describirlo como "un deporte que no consiste tan sólo en adquirir una cierta destreza, sino también en dominar el arte de ser un miembro de la sociedad bueno y honesto". También es importante mencionar que la practica ayuda al desarrollo tanto social, físico, psicológico, auto aprendizaje, formación del carácter, entre otros.

\section{Metodología}

La investigación realizada fue de tipo Cuasi-experimental, correlativa, acompañada de métodos teóricos, tales como: el histórico-lógico, el analítico- sintético, inductivo deductivo, los cuales permitieron realizar el análisis teórico del tema a desarrollar. Dentro de los métodos empíricos, se utilizó la observación y la medición, mediante el Test de Ozeretski Guillman, el cual comprende una batería de pruebas que permiten determinar la actitud motriz de los niños participantes, en relación con la coordinación dinámica general, coordinación ojo-mano y control postural y equilibrio.

La población fue integrada por 28 niños, con discapacidad intelectual, procedentes del Centro de Desarrollo Integral el Niño, Fundación Tierra Nueva, a los cuales se les aplicó el Test de Ozeretski Guillman en dos momentos pretest y postest. Los criterios de inclusión determinados para la selección de la muestra fueron:

- Presentar discapacidad intelectual

- Proceder del Centro de Desarrollo Integral el Niño, Fundación Tierra Nueva

- Estar autorizados para participar de la práctica deportiva del Karate Do.

Determinados los resultados del pretest se realizaron 4 semanas de intervención mediante la aplicación de un Programa de sesiones de Karate-Do, utilizando la técnica del Kihon. Posteriormente se realizó una segunda medición (postest), para valorar el desarrollo logrado en las habilidades siguientes: Coordinación óculo -manual, dinámica general, control postural y equilibro, analizándose los resultados mediante la escala de Likert.

El programa de intervención de ejercicios de Karate-Do basados en la técnica del Kihon para potenciar la habilidad motriz estuvo compuestos de los siguientes ejercicios, posiciones del Karate-Do (saludo, posiciones adelantadas, posiciones retrasadas, equilibradas), golpes ( técnica del cierre del puño, contracción, desplazamiento y movimiento de la cadera), bloqueos ( básicos), patadas (básicas).

Para la realización de los ejercicios se implementa una serie de reglas las que deben ser acatadas por los niños dentro de la clase:

- Todos ingresan con ropa deportiva adecuada para el desarrollo correcto de la clase.

- Los sujetos deben ingresar y salir de la clase realizando el saludo del Karate-Do y deben decir la palabra "Oss". 
- Todos los sujetos deben esperar primero que el docente realice la técnica para luego ellos realizarla.

- Los sujetos deben acatar las órdenes del docente.

- Para cada una de las técnicas a realizarse deben adoptar la postura correcta.

Además, se implementaron algunas indicaciones metodológicas para que la clase fuera desarrollada correctamente. Dentro de estas indicaciones se prestó especial interés a la demostración de cada una de las técnicas por parte del docente, ubicación de una cruz en el piso con cinta masking para facilitar el aprendizaje de los estudiantes, utilización de un cuadrado de 25 por $25 \mathrm{~cm}$ como medio de aprendizaje de una de las técnicas aprendidas, utilizándose diversos canales de comunicación como el gestual, visual y oral, existirá la corrección de ejecución y postura continuamente por parte del docente.

\section{Resultados.}

A continuación se presentan los resultados obtenidos de la investigación realizada.

Tabla No. 1. Caracterización y Distribución de Niños por Discapacidad

\begin{tabular}{cccc}
\hline \multicolumn{3}{c}{ discapacidad } & \\
\cline { 1 - 2 } Sexo & Discapacidad Intelectual & Sindrome de Down & \\
Femenino & 3 & 8 & 11 \\
Masculino & 10 & 7 & 17 \\
& 13 & 15 & 28 \\
\hline
\end{tabular}

Elaborado por Cuzco \& Rodríguez (2021)

Se observa que de una muestra del $100 \%$ de participantes en condiciones de discapacidad; 13 tienen Discapacidad Intelectual y 15 tienen Síndrome de Down de un total de 28 estudiantes del Centro de Desarrollo Integral el Niño Fundación Tierra Nueva.

Tabla 2. Correlaciones de Muestras Emparejadas T Student

\begin{tabular}{lccc}
\hline \multicolumn{3}{c}{ Correlaciones de muestras emparejadas } \\
\hline
\end{tabular}

Elaborado por Cuzco \& Rodríguez (2021)

Una vez analizado los datos y relacionados sus resultados pre test y post test bajo el instrumento estadístico de la T de Student en la coordinación óculo manual se observa 
resultados del Signo. (bilateral) de ,000, lo cual determina una diferencia significativa entre sus resultados siendo menor a ,005. Por otro lado, en la coordinación Dinámica General se observa resultados del Signo. (bilateral) de ,000, lo cual determina una diferencia significativa entre sus resultados siendo menor a ,005. De igual manera en el Control Postural y Equilibrio se observa resultados del Signo. (bilateral) de ,000, determinadose una diferencia significativa entre sus resultados siendo menor a ,005.

Tabla No. 3. Resultados de la aplicación del Test de Ozeretski

\begin{tabular}{|c|c|c|c|c|c|c|}
\hline \multirow[t]{2}{*}{$\mathrm{N}^{\mathrm{a}}$} & \multicolumn{2}{|c|}{ Coordinación Óculo Manual } & \multicolumn{2}{|c|}{$\begin{array}{c}\text { Coordinación Dinámica } \\
\text { General }\end{array}$} & \multicolumn{2}{|c|}{$\begin{array}{c}\text { Control Postural y } \\
\text { Equilibrio }\end{array}$} \\
\hline & pre-test & post-test & pre-test & post-test & pre-test & post-test \\
\hline $\mathbf{1}$ & 5 & 6 & 2 & 4 & 3 & 5 \\
\hline 2 & 3 & 7 & 4 & 8 & 4 & 7 \\
\hline 3 & 2 & 5 & 3 & 5 & 4 & 6 \\
\hline 4 & 8 & 10 & 6 & 8 & 5 & 8 \\
\hline 5 & 5 & 8 & 3 & 4 & 5 & 7 \\
\hline 6 & 2 & 3 & 0 & 0 & 2 & 4 \\
\hline 7 & 5 & 6 & 3 & 4 & 4 & 6 \\
\hline 8 & 6 & 8 & 4 & 8 & 5 & 7 \\
\hline 9 & 4 & 6 & 5 & 7 & 4 & 5 \\
\hline 10 & 3 & 5 & 1 & 3 & 5 & 8 \\
\hline 11 & 4 & 6 & 3 & 5 & 5 & 7 \\
\hline 12 & 6 & 10 & 5 & 7 & 7 & 9 \\
\hline 13 & 6 & 8 & 3 & 5 & 3 & 6 \\
\hline 14 & 6 & 9 & 6 & 8 & 4 & 6 \\
\hline 15 & 1 & 3 & 1 & 2 & 0 & 1 \\
\hline 16 & 3 & 4 & 2 & 4 & 0 & 3 \\
\hline 17 & 5 & 7 & 4 & 6 & 4 & 7 \\
\hline 18 & 3 & 5 & 5 & 7 & 6 & 8 \\
\hline 19 & 6 & 8 & 3 & 5 & 4 & 6 \\
\hline 20 & 4 & 7 & 3 & 5 & 4 & 7 \\
\hline 21 & 5 & 8 & 4 & 6 & 6 & 9 \\
\hline 22 & 5 & 7 & 3 & 5 & 2 & 5 \\
\hline 23 & 2 & 5 & 3 & 4 & 3 & 5 \\
\hline 24 & 4 & 5 & 1 & 3 & 0 & 4 \\
\hline 25 & 8 & 10 & 9 & 10 & 7 & 10 \\
\hline 26 & 5 & 6 & 0 & 2 & 3 & 5 \\
\hline 27 & 6 & 8 & 6 & 9 & 5 & 8 \\
\hline 28 & 0 & 4 & 5 & 7 & 3 & 5 \\
\hline TOTAL & 122 & 184 & 97 & 151 & 107 & 174 \\
\hline
\end{tabular}


Los resultados obtenidos de las tres pruebas del test de Ozeretski (Tabla3), demostraron un total de 122 en la coordinación óculo manual, luego de implementar las sesiones de Karate-Do utilizando la técnica de kihon durante 4 semanas los resultados aplicándolos en una segunda ocasión o postest se obtiene un total de 184, teniendo una diferencia significativa a favor del postest (,000), según se establece en la correlación de muestras emparejadas (Tabla 2).

Para el caso de la coordinación dinámica general, el test de Ozeretski en su aplicación inicial o pretest se obtuvo un valor de 97, mientras que es su segunda aplicación nos lanzó datos de 151, existiendo una mejora y siendo significativamente diferente a favor del postest (,000), según se establece en la correlación de muestras emparejadas (Tabla 2).

Por otra parte, con respecto a la prueba de control postural y equilibrio se obtuvo en la primera aplicación del pretest con un valor de 107, mientras que en su segunda aplicación o postest, dando un valor de 174 como una mejoría significativamente diferente a favor del postest (,000), según se establece en la correlación de muestras emparejadas (Tabla 2).

En sentido general se puede apreciar lo siguiente:

1. Quedó demostrado mediante la aplicación del T de Student, que al comparar los resultados derivados de la medición inicial y final existió una diferencia significativa en las medias de los niveles logrados en las habilidades motrices de los niños.

2. Se aprecia que el programa aplicado tuvo efectos muy positivos en el desarrollo de las habilidades motrices de los estudiantes con discapacidad intelectual, con especial interés en los niños con Síndrome de Down

\section{Conclusiones.}

A modo de conclusiones se determina lo siguiente:

- Se evidenció la efectividad del programa realizado mediante la práctica de KarateDo basado en la técnica del Kihon, como una alternativa viable para el proceso de desarrollo integral de los niños que presentan discapacidad intelectual, con énfasis en sus habilidades motrices.

- Quedó demostrado que existe una mejora significativa en el desarrollo de las habilidades específicamente en la coordinación dinámica general, coordinación óculo manual, control postural y el equilibrio en niños con discapacidad intelectual, lo cual influye positivamente en los procesos de inclusión y en su desarrollo integral.

Agradecimiento, Al grupo de investigación AFIDESA (Actividad Física, Deporte y Salud ) de la universidad de las Fuerzas Armadas ESPE. Y al Centro de desarrollo integral el Niño fundación tierra nueva por permitirnos realizar la investigación en su centro. 


\section{Referencias Bibliográficas}

AAIDD. (2010). CREENA. Obtenido de https://creena.educacion.navarra.es/web/necesidades-educativasespeciales/equipo-de-psiquicos/discapacidad-intelectualp/definicion-dediscapacidad-intelectual/

Arana, D. A. (2017). Desarrollo de las Habilidades Motrices de las personas con Discapacidad Intelectual atravez del proceso cognitivo. Madrid: Arte y Salud.

Arteaga, Ramírez, \& Luna. (02 de 2020). Las habilidades de coordinación visomotriz para el aprendizaje de la escritura. Obtenido de http://scielo.sld.cu/scielo.php?script=sci_arttext\&pid=S221836202020000100116\&lng=es\&nrm=iso\&tlng=es\#: :text=La\%20coordinaci\%C 3\%B3n\%20visomotriz\%20implica\%20actividades $\% 20 \mathrm{de} \% 20$ movimientos $\% 20 \mathrm{c}$ ontrolados\%2C\%20es\%20decir,\%2C\%20rasgar\%2C\%20pegar\%2C\%20etc.

Contreras. (2011). La coordinación dinámica general. Obtenido de https://www.efdeportes.com/efd157/la-coordinacion-dinamica-general.htm

Dojo Keiko Shin España. (2016). Beneficios del Karate Do. Obtenido de https://keikoshinspain.com/es/pre-karate-do

Guthrie. (2015). habilidad motriz . EFdeportes.

Karate blog. (2018). El karate-do y sus beneficios . Día mundial del karate: qué beneficios nos ofrece este deporte.

Luckasson y Cols. (2011). Discapacidad intelectual. Obtenido de http://maltratoinfantilydiscapacidad.es/wpcontent/uploads/2015/06/Definiciones.pdf

Lucea, J. D. (1999). La enseñanza y aprendizaje de las habilidades y destrezas motrices básicas (Vol. 133). Inde.

Molina. (2011). COORDINACIÓN DINÁMICA GENERAL. Obtenido de https://www.efdeportes.com/efd157/la-coordinacion-dinamica-general.htm

ONU. (2018). Definición de discapacidad. Obtenido de https://www.un.org/esa/socdev/enable/documents/tccconvs.pdf

Rigal. (2006). DEFINICION DE COORDINACION. Juego y Deporte . Obtenido de https://www.juegoydeporte.com/educacion-fisica/coordinacion-muscular-omotora/

Schuba, \& Hafelinger . (2010). Revisión conceptual y tipología de la coordinación motriz. Obtenido 
https://www.efdeportes.com/efdeportes/index.php/EFDeportes/article/view/2047 $/ 1241$

Soto. (11 de 01 de 2019). Artes marciales: Karate paralímpico. Obtenido de https://soloartesmarciales.com/blogs/news/artes-marciales-karate-paralimpico

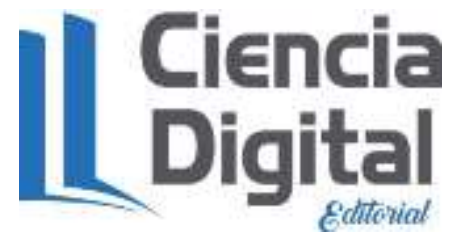


PARA CITAR EL ARTÍCULO INDEXADO.

María Angelica, C. Y., Solange Stephanie, R. M., \& José Guillermo, C. M. (2021). La práctica de karate-do y la habilidad motriz en niños con discapacidad intelectual.

Explorador Digital, 5(3), 126-138. https://doi.org/10.33262/exploradordigital.v5i3.1791

\section{Ciencia \\ Digital \\ Edtorial}

El artículo que se publica es de exclusiva responsabilidad de los autores y no necesariamente reflejan el pensamiento de la Revista Explorador Digital.

El artículo queda en propiedad de la revista y, por tanto, su publicación parcial y/o total en otro medio tiene que ser autorizado por el director de la Revista Explorador Digital.
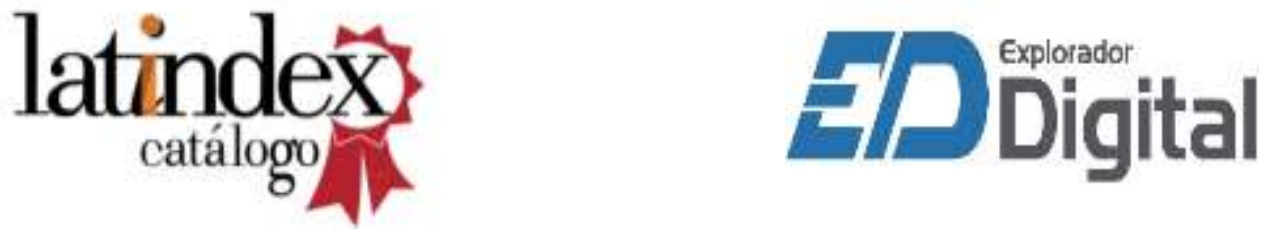